\title{
A Link Quality Estimator for Power-Efficient Communication over On-Body Channels
}

\author{
Mónica Vallejo \\ Departamento de Energía Eléctrica y Automática \\ Facultad de Minas, Universidad Nacional de Colombia \\ Medellín, Colombia \\ Email: mavallejov@unal.edu.co
}

\author{
Joaquín Recas, José L. Ayala \\ Department of Computer Architecture and Automation \\ Complutense University of Madrid \\ Madrid, Spain \\ Email:jrecas@fdi.ucm.es,jayala@fdi.ucm.es
}

\begin{abstract}
The human body has an important effect on the performance of on-body wireless communication systems. Given the dynamic and complex nature of the on-body channels, link quality estimation models are crucial in the design of mobility management protocols and power control protocols. In order to achieve a good estimation of link quality in WBSNs, we combine multiple body-related factors into a model that includes: the transmission power, the body position, the body shape and composition characteristics and the received signal strength indicator (RSSI) as an indicator of link quality. In this paper, we propose the Anfis Link Quality Estimator (A-LQE) that has been trained with RSSI values measured at different transmission power levels in a sample of 37 human subjects. Once the accuracy and reliability of our proposed model have been analysed, we apply the model to adapt the transmission power to the link characteristics for energy optimization. The obtained average energy savings reach the $26 \%$ in comparison with the maximum transmission power mode.
\end{abstract}

Keywords-WBSNs; on-body channels; link quality estimator; transmission power control; energy savings

\section{INTRODUCTION}

The increasing use of wireless networks and the constant miniaturization of electrical devices have boosted the development of Wireless Body Sensor Networks (WBSNs) as a special case of Wireless Sensor Networks (WSNs). In these networks, various sensors are attached on clothing, on the body, or even implanted under the skin. The wireless nature of the network and the wide variety of sensors offer numerous new, practical and innovative applications to improve health care and Quality of Life, or to track the performance of professional sportsmen. The sensors of a WBSN can measure, for example, the heartbeat, the body temperature, or record a prolonged electrocardiogram [1].

In a common WBSN scenario, the user is fully functional and develops a physical activity with regular exercise and movement. The mobility of the user causes variations in the separation and orientation of the antennas of the nodes. This fact can lead to changes in the quality of the links, affecting their reliability [2], [3]. Voluntary and involuntary movements cause shadowing, which affects the performance of the links; moreover, the shadow effect due to different body shapes has much influence in the path loss and in the distorted received signal [4].

Given the dynamic and complex nature of the on-body channels, the challenge is to maintain a good quality of the link between sensor nodes while extending the network lifetime. The use of a fixed transmission power can be inadequate since it results in an unnecessary energy waste (at high power values) or in a reduced reliability and increased retransmissions (at low power values). The transmission power control techniques allow to dynamically adjust the power levels according to the changing conditions of the links. Therefore, these techniques allow to meet the reliability constraints of the links, while saving at the same time the operational energy in sensor nodes, which are batteryconstrained devices.

The transmission power control techniques require to quantify the quality of the wireless links with the purpose of modifying the transmission power level in an energyefficient fashion, according to the current state of the link. The methods that estimate the wireless channel quality are called Link Quality Estimators (LQEs). Several LQEs have been reported in the literature for WSNs; these methods are typically based on the reading of hardware indicators and/or in received packet count [5], [6] computed over a run time window. Longer time windows contribute to improve the accuracy of the prediction, but however they also increase the energy consumption. Considering the special dynamics in WBSNs, there is clearly a need for LQE models that allow not only estimate but also to anticipate the run-time behavior of the on-body links with the minimum of memory requirements and traffic overhead.

Given the the complexity to find an exact analytical formula to prediction model of the RSSI, in this paper we propose the Adaptive Neuro Fuzzy Inference System (ANFIS) Link Quality Estimator (A-LQE), a model that allows to predict on-body link quality variations, by means of the Received Signal Strength Indicator (RSSI), for dynamic adjustment of the transmission power. We propose the use of this model based on Artificial Neural Network (ANN) because it combines the advantages of empirical 
and deterministic models, it has the flexibility to adapt to different environments and also, it includes several effects inherent to measurement data [7].

The A-LQE model combines parameters related with the sensor node location, the transmission power levels available in the radio, as well as the movement, shape and composition of the human body. The A-LQE model has been built and trained off-line with RSSI values measured in an experimental sample of 37 human subjects at different transmission power levels. Hence in our model, as opposed to previous approaches, the sensor nodes are not committed to collect in run time a huge amount of information from received packets. In contrast, our model only requires some constant parameters and the movement detection based on low overhead accelerometry.

Our main contributions are:

- An experimental methodology for the characterization of channel quality in biological subjects.

- A detailed analysis about the real impact of the features of the shape and body composition in the estimation of the link quality.

- A predictor model for RSSI variations in on-body links based on ANFIS. This model combines information from several parameters related with human body characteristics and it has been exploited for dynamically adjusting the transmission power in order to obtain energy savings.

- The classification of the experimental human sample according to their behavior in terms of the transmission power.

This article is organized as follows. Section II briefly introduces the related works in the LQEs. Section III presents the experimental setup used for the characterization of onbody channel quality in human subjects. In Section IV, we present our A-LQE and a brief theoretical background for this heuristics; after that, we discuss how this model can be used for adjusting the transmission power in an energyefficient way. Finally, Section V draws the conclusions and future work.

\section{RELATED WORK}

In wireless sensor networks, the packet loss and energy consumption depend critically on the quality of the network's wireless links. Given the importance of obtaining link quality estimators in wireless networks, several proposals have been reported in the literature based on a single or combination of different metrics [5], [6], [8]. The physical layer metrics used for link estimation, and provided by the radio hardware are: Received Signal Strength Indicator, Link Quality Indicator and Signal-to-Noise Ratio. On the other hand, the Packet Reception Rate and Packet Loss Rate are the most common link layer metrics and these are always calculated based on a given number of packet transmissions. Depending of the metrics used, some these approaches can involve high computation overhead and higher memory footprint on sensor nodes.

In the WBSNs case, the human body has an important effect on the performance of the communication, it detunes and distorts the radiation pattern of the antenna, and it introduces attenuation due to absorption of power in the tissue or propagation blockage [9], [10], which is dissipated as heat. Di Franco et al [4] showed that the shape of human body is an additional critical parameter in channel models. However, despite of these evidences, until now none of the LQE approaches presented in the literature have considered including some property that takes into account the effects of operating near the human body.

We propose a model that combine parameters related with the sensor node location, the transmission power levels, the movement, shape and composition of the human body and it allows to predict on-body link quality variations by means of the RSSI, for dynamic adjustment of the transmission power.

\section{EXPERIMENTAL SETUP}

Along the presented experimental work, we seek to establish an analytical model that predicts the RSSI metric in terms of several input parameters. The devised model can be used to dynamically adjust the transmission power so that the minimum level that ensures a good link quality between a pair of nodes can be selected. With this experimental profiling, we are interested in the research about the real impact of body movements, body shape and body composition as input parameters in the RSSI model. In this paper, we follow a similar experimental methodology to [11], but we extend the sample group to a larger population of 37 people with different physical characteristics, what allows to extract several interesting conclusions.

In our experiments, we use the sensor nodes Shimmer [12]. The Shimmer node is equipped with an ultra-lowpower 16-bit microcontroller (TI MSP430) that runs at a maximum clock frequency of $8 \mathrm{MHz}$ and includes $10 \mathrm{~KB}$ of RAM and $48 \mathrm{~KB}$ of Flash. This platform has also two radio links, IEEE 802.15.4-compliant CC2420 transceiver [13]. The transceiver CC2420 has a sensitivity threshold of -94 $\mathrm{dBm}$ and provides eight programmable power transmission levels, from maximum level at $0 \mathrm{dBm}$ with a current consumption of $17.4 \mathrm{~mA}$, to minimum level at $-25 \mathrm{dBm}$ with current consumption of $8.5 \mathrm{~mA}$ (see Table I). This sensor node also uses a GigaAnt Rufa SMD antenna operating at $2.4 \mathrm{GHz}$ with omnidirectional radiation pattern. We have ported FreeRTOS for this platform, which is a portable, open source, and hard real-time mini kernel that includes support for the microcontroller and the IEEE 802.15.4-compliant radio chip.

The nodes were placed on the subjects' bodies describing a star topology, with the coordinator placed in the waist (just over the navel), and the node sensors in the right arm 
Table I

OUTPUT POWER SETTINGS AND TYPICAL CURRENT CONSUMPTION OF SHIMMER NODE

\begin{tabular}{|c|c|c|c|c|c|c|c|c|}
\hline TxPower $[\mathrm{dBm}]$ & 0 & -1 & -3 & -5 & -7 & -10 & -15 & -25 \\
\hline $\mathrm{I}[\mathrm{mA}]$ & 17.4 & 16.5 & 15.2 & 13. & 12.5 & 11.2 & 9.9 & 8.5 \\
\hline
\end{tabular}

(link L1) and in the right knee (link L2) as shown in Fig 1. For the sake of clarity, we have considered a data rate and packet size fixed. The evaluation of how these parameters can affect the communication is considered as future work. The measurements were repeated to 7 transmission power levels for every subject. We will not consider the minimum transmission power level $(-25 \mathrm{dBm})$ due as the associated failure rate in transmission is unacceptable for our experiments. All the results were taken in controlled conditions to minimize the effect of interfering EM-waves (WiFi, 3G, solar radiation,etc.)

For every subject, we took anthropometric measurements and body composition. The anthropometric parameters are shown in Fig. 1. These parameters were selected because they are directly related with the locations of the nodes and the links of interest. On the other hand, the measures of body composition were done using the Tanita tetrapolar foot-to-foot bioelectrical impedance analyzer, Model BC601 [14]. These body composition parameters include: body fat, muscle mass, bone mass, body mass index, total body water and the levels of body fat percentage and muscle mass of each segment (right/left arm and right/left leg).

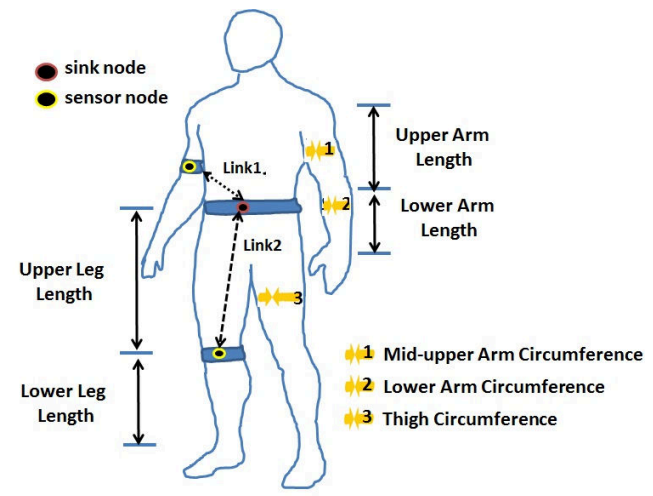

Figure 1. Node location and anthropometric measurements

We planned two experimental scenarios to investigate the temporal variations in the quality of two links in stationary positions:

- Scenario 1: the subject sat on a chair performed five movements of the arms (Link 1): 1) hands on thighs, denoted as L1/P1; 2) arms crossed, L1/P2; 3) arms extended forward, L1/P3; 4) arms extended up, L1/P4; and 5) arms extended to both sides, L1/P5.

- Scenario 2: the subject sat on a chair performed four movements of the legs (Link 2): 1) leg in $90^{\circ}$ angle with the body, L2/P1;2) left leg crossed over the right knee, L2/P2; 3) right leg crossed over left knee, L2/P3; and 4) leg extended forward, L2/P4.

Figure 2 shows the box plot of the average RSSI of the experimental data for every power transmission level and for every movement considered in scenarios 1 and 2 . We can observe that the most favourable positions are L1/P1 in scenario 1 , and L2/P1 and L2/P2 in scenario 2 because, independently of the power transmission level used, RSSI is always above of the sensitivity threshold (dotted line in the figure) and there are no packets loss. This fact can be explained because the sensor nodes have direct line of sight in these configurations and are located at short distance.
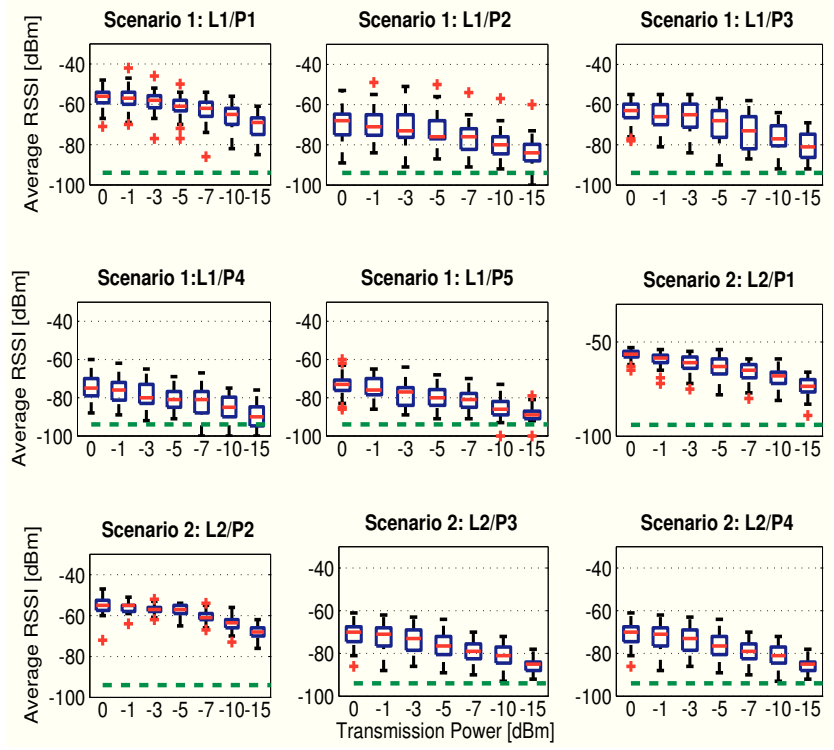

Figure 2. Box plot of experimental average RSSI for the considered movements in Scenario 1 and 2. The sensitivity threshold is shown with a dotted line

For the positions $\mathrm{L} 1 / \mathrm{P} 2, \mathrm{~L} 1 / \mathrm{P} 3$ in scenario 1 and $\mathrm{L} 2 / \mathrm{P} 3$, $\mathrm{L} 2 / \mathrm{P} 4$ in scenario 2 , we can observe that at lower power transmission levels, the RSSI is very close to the threshold, it causing a large percentage of packet losses. The most critical positions are L1/P4 and L1/P5 in scenario 1 . For both cases, we can observe that RSSI is very close to the threshold and drops below to the threshold at power transmission levels from $-10 \mathrm{dBm}$ to $-15 \mathrm{dBm}$ with large percentage of packet losses. The shadow area is wider as compared with the other two positions, hence this fact has a greater impact when lower power transmission levels are used. This preliminary study explains why several positions and links considered in the experiments will require higher power transmission level to meet the sensitivity threshold. This fact will be consequently captured by the model presented in section IV. 


\section{ANFIS LINK QUALITY Estimator (A-LQE)}

Based on our empirical study, a model based on ANFIS is proposed for each of the two links that have been experimentally characterized. The A-LQE allows to predict the onbody link quality variations (in terms of the RSSI parameter) in order to dynamically adjust the transmission power and, in this way, extend the battery life of the sensors node. These models involve the interaction of multiple input parameters related with the sensor node location, the transmission power levels available in the radio, as well as the movement, shape and composition of the human body, and provide as output the RSSI of the link. In order to build reasonable models for prediction, we follow a two-phase approach: in the first step, we carefully selected the set of uncorrelated variables that can serve as input parameters for the model; in the second step, we tested various combinations of input variables to find the most accurate model with the lowest complexity of the neural network.

In this section, we give a brief theoretical background to this ANFIS heuristics. After that, we present the input variable selection process; then, we show the structure of our resulting model, and finally we discuss how this model can be used for adjusting the transmission power in an energyefficient way.

\section{A. Brief Overview of ANFIS}

Artificial neural network and Fuzzy logic are two important methods of artificial intelligence for modeling nonlinear problems. ANFIS is a multilayer feed-forward network consisting of nodes and directional links, which combines the learning capabilities of neural networks and knowledge representation and inference capabilities of fuzzy logic [15].By using the input-output data set, ANFIS creates a Fuzzy Inference System whose membership function parameters are adjusted using a back propagation algorithm alone or a combination of a back propagation algorithm with a least squares method (hybrid learning method), this allows the fuzzy systems to learn from the data being modeled to achieve a desired input-output mapping [16], [17]. There are two types of fuzzy inference systems: Mamdani-type and Sugeno-type [15], [18]. The Sugeno system is more compact and computationally more efficient; therefore, this one is typically used for constructing ANFIS models. The whole conceptual and mathematical details of ANFIS are not presented in this paper but we invite readers to review the documents which are cited in this section for further information.

\section{B. Input Variable Selection}

The Input variable selection is an important part in the construction of our nonlinear model. Incorporating only the uncorrelated variables provides a simpler, more useful, and more reliable model [19]. Therefore, our purpose in this stage is to remove this redundancy created by correlations among the variables.

An overall of 12 and 13 variables are available as input parameters for the models of Link 1 and Link 2 respectively. The Pearsons correlation coefficient was used to verify correlations between anthropometric variables and body composition. A probability value of $\mathrm{p}<0.05$ was considered to indicate statistical significance. Both correlation coefficient $(r)$ and P-value have been calculated for the body parameters using the software Minitab-16 for both link models.

From this analysis, we grouped the variables in three and five groups for the Link 1 and Link 2, respectively. In particular, we found that variables such as total body fat, body fat of right leg (or right arm), total body water and body mass index present a significant correlation $(r>0.9)$ among them; muscle mass, bone mass and muscle mass of right leg (or right arm ) are strongly correlated as well $(r>0.9)$. On the other hand, the anthropometric variables of Link 1 show a significant correlation between them (length and circumference) while there are not correlation for the Link 2. Taking as a selection criteria the highest correlation with the measured RSSI, we selected the following variables for each one of the link models:

- Input Variables for Link 1: Body Fat, Upper Arm Circumference and Lower Arm Length.

- Input Variables for Link 2: Body Fat Right Leg, Bone Mass, Upper Leg Length, Thigh circumference and Lower Leg Length.

Then, our reduced models include five and seven input variables for the Link 1 and Link 2, respectively, including the transmission power and body position.

We are also interested in assuring that the selected input variables are meaningful and descriptive of the output variable (RSSI), i.e., that there are strong relationships among the parameters and the target variable. For this, we constructed ANFIS models for various combinations of input variables and then we chose the one with the best performance (lowest error). For this purpose, we have developed a selection method [19] in Matlab, which starts with a simple model and gradually increases the number of variables. This method reports when a model achieves the lowest Root Mean Squared Error (RMSE) of the testing and training set.

\section{ANFIS model parameters}

The different ANFIS models tested for both links were developed using MATLAB Fuzzy Logic Toolbox. The Sugenotype fuzzy inference system was used to obtain a concise representation of the system's behavior with a minimum number of rules. We evaluated several types of membership functions, such as triangular, trapezoidal, bell and gaussian shapes, with two, three and five functions for each input variable. Also, we tried the both fuzzy inference system generation methods (grid partition and subtractive clustering) 
and the hybrid learning algorithm, which combines the leastsquares method and the back-propagation gradient descent method for emulating a given training data set. Finally, we chose a linear membership function for the RSSI output variable.

The ANFIS models were trained with 1036 vectors of input data, collected during the experimental work. 724 vectors $(70 \%)$ were randomly chosen for training set, 156 (15\%) vectors for testing set, and the other $156(15 \%)$ vectors for validation set. The generalization capability of the model is assured by the proper selection of a large training set. Also, 100 epochs and a training error tolerance of 0.0001 were specified for the training process to assure the achievement of the minimum error tolerance.

Table II shows a comparison of the obtained ANFIS models for Link 1 and Link 2. These models include from one to four input variables. The criteria used for evaluating the network performance are the Root Mean Squared Error (RMSE), Absolute Fraction of Variance $\left(R^{2}\right)$ and Mean Absolute Error (MAE). Network performance is assured for high values of $R^{2}$, and low values of MAE and RMSE. Detailed definitions of these criteria can be found in [20].

From the values in Table II, we can see how the subject position has a strong impact on the accuracy of the model, even more than the Transmission Power (PTx). So for the Link 1, if we analyze the first two models with Ptx and body position (BPosition) as unique inputs, we can see how the second model improves the performance metrics $\left(R^{2}=0.61\right.$, RMSE $=8.49$, and MAE =7) with respect to the first model $\left(R^{2}=0.44\right.$, RMSE=9.34, and MAE =7.80). For the third model, we note that the combination of both inputs (Ptx and BPosition) introduces a maximum improvement in $R^{2}$ of 0.34 and a reduction of RMSE and MAE of almost 2 units with respect to the first two models. Finally, the comparison of the forth and fifth models proves once more the significance of the body position parameter in the model.

Table II

COMPARISON OF ANFIS MODELS FOR BOTH LINKS WITH TRAINING DATA SET

\begin{tabular}{|c|c|c|c|c|c|}
\hline Link & Model & Inputs Name & RMSE & R2 & MAE \\
\hline \hline & 1 & Ptx & 9.34 & 0.44 & 7.80 \\
& 2 & BPosition & 8.49 & 0.61 & 7.00 \\
L1 & 3 & Ptx/BPosition & 6.45 & 0.78 & 5.11 \\
& 4 & Ptx/BFTotal/CArm & 8.94 & 0.52 & 7.46 \\
& $\mathbf{5}$ & Ptx/BPosition/BFTotal/CArm & $\mathbf{6 . 1 5}$ & $\mathbf{0 . 8 1}$ & $\mathbf{4 . 8 1}$ \\
\hline \hline \multirow{4}{*}{ L2 } & 1 & Ptx & 8.21 & 0.54 & 6.83 \\
& 2 & BPosition & 7.23 & 0.66 & 5.92 \\
& $\mathbf{3}$ & Ptx/BPosition & $\mathbf{4 . 8 9}$ & $\mathbf{0 . 8 6}$ & $\mathbf{3 . 7 9}$ \\
& 4 & Ptx/LLeg & 8.10 & 0.53 & 6.70 \\
& $\mathbf{5}$ & Ptx/BPosition/LLeg & $\mathbf{4 . 9 3}$ & $\mathbf{0 . 8 5}$ & $\mathbf{3 . 8 2}$ \\
\hline \hline
\end{tabular}

After this analysis, we can conclude that the most accurate model for Link 1 (high $R^{2}=0.81$, low $\mathrm{RMSE}=6.15$, and low MAE=4.81) is the model 5, it includes Transmission Power, Body Position, Body Fat Total (BFTotal) and Cir- cumference Arm (CArm) as input parameters. The same analysis can be done for the models of Link 2. From this analysis, we can conclude that there are two good models for the Link 2: model 3 with two inputs: Transmission Power and Body Position and the model 5 with three inputs: Transmission Power, Body Position and as body parameter, the Leg Lower Length (LLEG).

It is also important to note from Table II that the sensor node placement on the body, plays an important role in the A-LQE model. Thereby, the analysis of RMSE values shows that for Link 1, where the receiving node is located in the arm, the RSSI shows more dispersive behavior due to higher probability of involuntary movements than Link 2, where there is less scattering of data.

\section{ANFIS model structure}

The architectures of the developed A-LQE models for every link are shown in Fig. 3. These models are composed of five interconnected layers. The layer 1 consists of adaptive nodes that evaluate the fuzzy membership grade defined for the inputs. The layer 2 consists of fixed nodes that represent the firing strength of each fuzzy rule. The layer 3 consist of fixed nodes whose output is the normalized firing strength of each rule. The layer 4 consists of adaptive nodes whose output is the product between degrees of activation normalized by the individual output of each rule. Finally, the layer 5 consists of a single node that computes the overall output of the system as the sum of all individual incoming signals [15], [21].

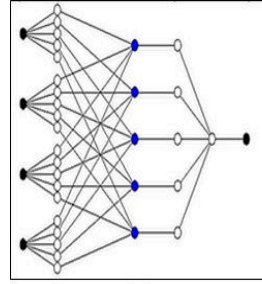

(a)

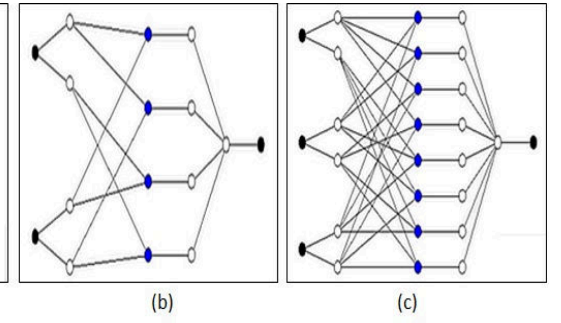

Figure 3. ANFIS structure for Links. (a) Structure for Link1; (b) Structure 1 for Link2; (c) Structure 2 for Link2

For the A-LQE model of Link 1, which is shown in Fig. 3a, we used a fuzzy subtractive clustering algorithm to partition the input space and construct a compact fuzzy-based rule to predict the data behavior properly. As aforementioned, this model has four input parameters: Transmission Power, Body Position, Body Fat Total and Circumference Arm, with five membership functions of Gaussian type for every input variable, five rules and one linear output. For A-LQE models of Link 2, which are also shown in Fig. 3b and Fig. 3c, we used a fuzzy c-means clustering algorithm. The first model has only two input parameters: Transmission Power and Body Position with two membership functions of trapezoidal type for every input variable, four rules and one 
linear output; the second model has three input parameters: Transmission Power, Body Position and Leg Lower Length, with two membership functions of triangular type for every input variable, eight rules and one linear output.

\section{E. Results}

Table III shows the results of our A-LQE models for both links when these are evaluated against the testing dataset. The validation of the predictive accuracy of the models is analysed through RMSE, MAE and Average Percentage Error (APE). As can be seen, the A-LQE models produce satisfactory results with a low APE of $6.01 \% 5.56 \%$ and $5.24 \%$ for Link 1 and two models of Link 2 respectively. The model of three inputs for Link 2 gives a lower APE than the other model, by this reason, henceforth we focus on this model. Therefore, the A-LQE models can be used to predict the RSSI in on-body channels and, thereby, perform a dynamic adjustment of the transmission power. Hence in our model, as opposed to previous approaches where a reactive behavior is proposed, the sensor nodes are not committed to collect in run time a huge amount of information from received packets. In contrast, our model only requires some constant parameters and the movement detection based on low overhead accelerometry.

Table III

COMPARISON OF A-LQE MODEL FOR BOTH LINKS WITH TESTING DATA SET

\begin{tabular}{|c|c|c|c|c|}
\hline Link & Inputs Name & APE $[\%]$ & RMSE & MAE \\
\hline \hline L1 & Ptx/BPosition/BFTotal/CArm & $\mathbf{6 . 0 1}$ & $\mathbf{5 . 5 1}$ & $\mathbf{4 . 3 9}$ \\
\hline \hline L2 & Ptx/BPosition & 5.56 & 4.71 & 3.73 \\
\hline \hline L2 & Ptx/BPosition/LLeg & $\mathbf{5 . 2 4}$ & $\mathbf{4 . 5 7}$ & $\mathbf{3 . 5 8}$ \\
\hline \hline
\end{tabular}

\section{F. Computational Complexity Analysis of A-LQE models}

The complexity of a general ANFIS Network can be analysed from Table IV as follows:

Table IV

COMPLEXITY OF ANFIS NETWORK

\begin{tabular}{|c|c|c|c|}
\hline Layer \# & Type & \# Nodes & \# Param \\
\hline \hline$L_{0}$ & Inputs & $\mathrm{n}$ & 0 \\
\hline$L_{1}$ & Values & $(n \cdot p)$ & $3 \cdot(n \cdot p)=|S 1|$ \\
\hline$L_{2}$ & Rules & $p^{n}$ & 0 \\
\hline$L_{3}$ & Normalize & $p^{n}$ & 0 \\
\hline$L_{4}$ & Lin. Funct. & $(n+1) \cdot p^{n}$ & $|S 2|$ \\
\hline$L_{5}$ & Sum & 1 & 0 \\
\hline \hline
\end{tabular}

$\mathrm{S} 1$ and S2 are the sets of parameters used by ANFIS. S1 represents the fuzzy partitions used in the rules "left hand side" for tuning by back-propagation

$$
\begin{aligned}
S 1=\{ & \left\{a_{11}, b_{11}, c_{11}\right\},\left\{a_{12}, b_{12}, c_{12}\right\}, \ldots, \\
& \left.\left\{a_{1 p}, b_{1 p}, c_{1 p}\right\},\left\{a_{n p}, b_{n p}, c_{n p}\right\}\right\}
\end{aligned}
$$

while S2 represents the coefficients of the linear functions in the rules "right hand side" for tuning by Takagi-Sugeno

$$
S 2=\left\{\left\{c_{10}, c_{11}, \ldots, c_{1 n}\right\}, \ldots,\left\{c_{p^{n} 0}, c_{p^{n}}, \ldots, c_{p^{n} n}\right\}\right\}
$$

ANFIS uses a two-pass learning cycle. During the forward pass, S1 is fixed and S2 is computed using a Least Square Error algorithm (off-line learning). During the backward pass, $\mathrm{S} 2$ is fixed and $\mathrm{S} 1$ is computed using a gradient descent algorithm (back-propagation).

Besides the optimal characteristics of ANFIS in terms of smoothness (due to the Fuzzy Control interpolation) and adaptability (due to the Neural Network Backpropagation), it exhibits strong computational complexity restriction due to the size of S1 and S2 sets. We have applied several changes to decrease this complexity: i) reduce the subset of inputs; ii) use "don't care" values in rules to reduce the number of nodes per layer. These characteristics are also important for an on-line implementation on a sensor node because these models do not require a larger training sets. Moreover, the complexity of the ANFIS is exhibit during the training phase (performed once), while the prediction capabilities can be applied in real-time.

In our experiments, the parameters of the models are shown in Table V.

Table V

PARAMETERS OF A-LQE MODELS

\begin{tabular}{|c|c|c|c|}
\hline A-LQE models & Nodes & Lineal/No-Lineal Parameters & Rules \\
\hline \hline Ptx/BPosition/BFTotal/CArm & 57 & 65 & 5 \\
\hline \hline Ptx/BPosition/LLeg & 34 & 50 & 8 \\
\hline \hline
\end{tabular}

Table VI compares the number of mathematical operations required by each one of the A-LQE models.

Table VI

COMPARISON OF THE NUMBER OF OPERATIONS FOR A-LQE MODELS

\begin{tabular}{|c|c|c|c|c|}
\hline A-LQE models & Sums & Products & Divisions & Exponentials \\
\hline \hline Ptx/BPosition/BFTotal/CArm & 40 & 120 & 25 & 20 \\
\hline \hline Ptx/BPosition/LLeg & 56 & 32 & 20 & 0 \\
\hline \hline
\end{tabular}

\section{G. Case Study}

In this section, a case study is presented to show how our policy of transmission power control (TPC), based on the proposed A-LQE models for Link 1 and Link 2, works. In particular, four experimental human subjects out-of-thesample used for the training of the model are considered to show how the hybrid TPC approach proposed in Fig. 4 can be applied to achieve an energy-aware data transmission. This hybrid TPC approach consists of two main blocks: the A-LQE model and the TPC block. We remind that the A-LQE models only need as input variables an initial 
transmission power value, the body position detected by accelerometry with low-complexity and low overhead, and some constants of body parameters (Body Fat Total and Circumference Arm for the Link 1 and Leg Lower Length for the Link 2). The TPC block adjusts the transmission power to the minimum value to assure that the RSSI value does not drop below a threshold (which has been fixed to $-85 \mathrm{dBm}$ as proposed in [11]).

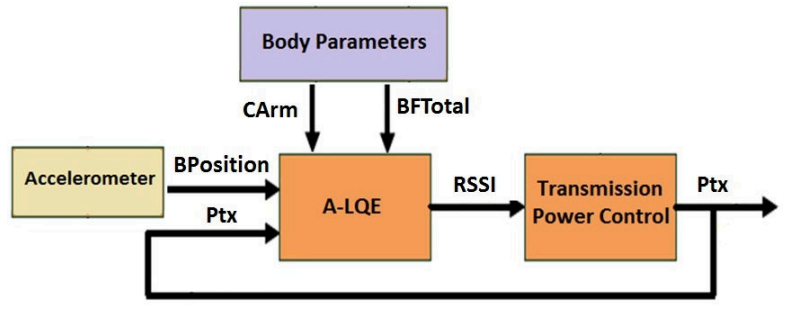

Figure 4. Architecture for hybrid TPC approach

The policy implemented to Transmission Power Control block, shown in the Fig. 4, is depicted in algorithm 1. The range of RSSI values is divided in four zones according to three constants: Tmin $=-85 \mathrm{dBm}$, Tmaxlower $=-80$ and Tmaxupper $=-68 \mathrm{dBm}$, choice of these constants have been done from the experimental results (see Fig. 2).

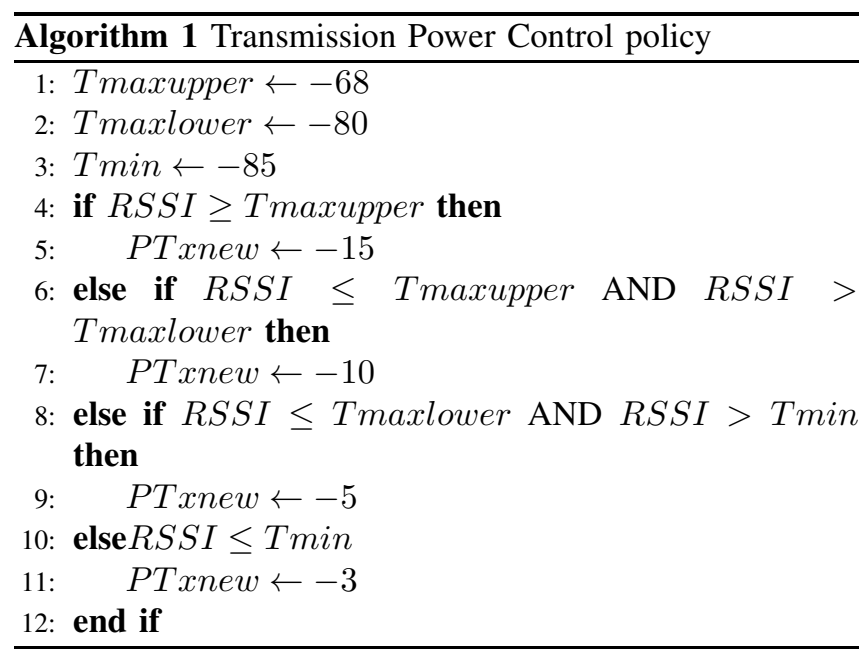

The experimental human subjects have been required to perform the following sequence of movements that simultaneously combine positions of the scenario 1 and 2 : $\mathrm{L} 1 / \mathrm{P} 1+\mathrm{L} 2 / \mathrm{P} 4, \mathrm{~L} 1 / \mathrm{P} 2+\mathrm{L} 2 / \mathrm{P} 3, \mathrm{~L} 1 / \mathrm{P} 3+\mathrm{L} 2 / \mathrm{P} 1, \mathrm{~L} 1 / \mathrm{P} 4+\mathrm{L} 2 / \mathrm{P} 2$ and $\mathrm{L} 1 / \mathrm{P} 5+\mathrm{L} 2 / \mathrm{P} 1$. The sequence begins with a first movement set $(\mathrm{L} 1 / \mathrm{P} 1+\mathrm{L} 2 / \mathrm{P} 4)$ at maximum transmission power; then the A-LQE models (of each link respectively) predict a RSSI value, finally, TCP block provides the adjusted transmission power level which, in its turn, generates a new cycle of outputs update in the case that current transmission power level or position have changed. The results of this section are shown in two parts: RSSI Predicted results and Energy Consumption results.

1) RSSI Predicted results: Fig. 5 shows the predictions obtained with the trained A-LQE models for every human subject of the case of study. For each link, all positions at all transmission power levels are considered. From these results, the Average Percentage Error obtained for each subject is $5.6 \%, 6.3 \%, 5.8 \%$ and $7.2 \%$ for link 1 and $3.9 \%, 6 \%, 8.4 \%$ and $4.2 \%$ for link 2 respectively. Even though this error value is less than $10 \%$ it seems that the RSSI trace predicted has troubles to follow the extreme values. Therefore, we try to compensate this fact with our conservative policy proposed for the TCP block.

2) Energy Consumption results: For the four cases of study considered in this section, we got from our approach a set of transmission power levels of $-5 \mathrm{dBm},-10 \mathrm{dBm}$ and $15 \mathrm{dBm}$ for the movement sequence, meaning that there was not any critical case for which the RSSI value dropped below the threshold. The Energy Consumption results are shown for three different configurations: transceiver configured to transmit at Maximum Power $0 \mathrm{dBm}$, at Minimum Power $25 \mathrm{dBm}$ and at Optimum Power, which corresponds with the hybrid TPC approach that dynamically selects the minimum transmission power that assures the quality of the link for every phase of the complex movement.
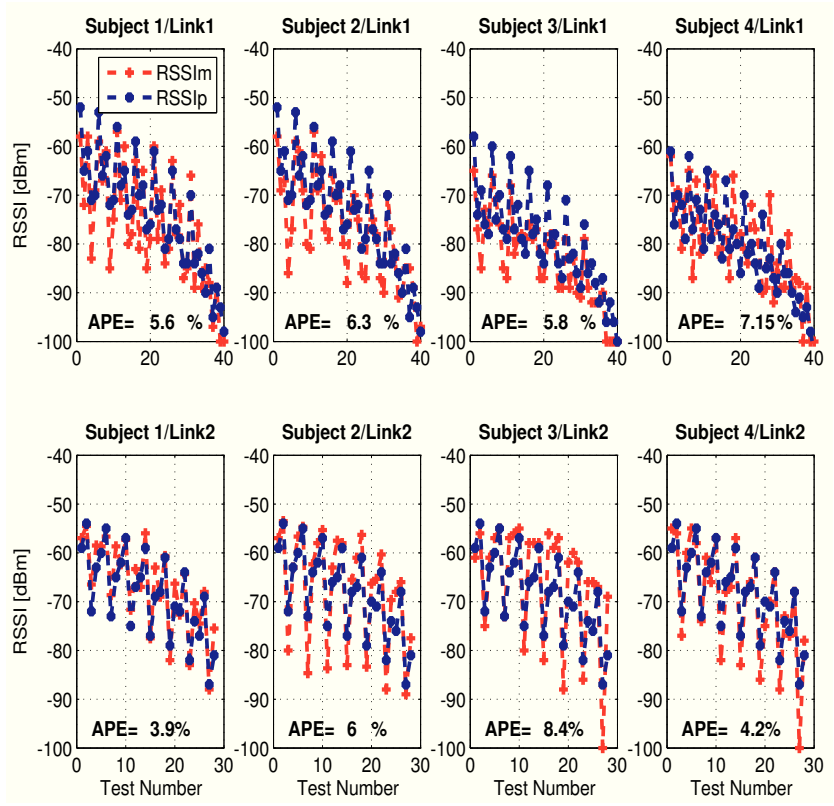

Figure 5. RSSI Predicted results for cases of study. RSSIm=measurement value, RSSIp=predicted value.

From the results shown in the Table VII, we observe that when the hybrid TPC approach is applied, the radio transceiver reduces the average energy consumption from $57.4 \mathrm{~J}$ to $42.38 \mathrm{~J}$ (an average reduction of $26.5 \%$ of the total energy for all subjects in comparison with the maximum 
transmission power mode). From the table we observed that the energy savings obtained with the hybrid TPC approach vary for each subject, but even in the worst cases (subject 4), we achieve significant savings of $23.3 \%$. Although our hybrid TPC approach obtains promising results, the TPC block can be improved to avoid the continuous change in the transmission power a same position, due to the feedback loop of the algorithm. However, in our experimental setup there is no overhead due to this fact.

Table VII

ENERGY CONSUMPTION OF SUBJECTS WITH HYBRID TPC APPROACH

\begin{tabular}{|l|c|c|c|c|}
\hline \multirow{2}{*}{ Subjects } & \multicolumn{3}{|c|}{ Energy consumption [J] } & \multirow{2}{*}{ Energy saving [\%] } \\
& Max Power & Min Power & TPC approach & \\
\hline \hline Subject 1 & 57.5 & 45.9 & 39.4 & 31.3 \\
\hline \hline Subject 2 & 58.8 & 43.2 & 42.8 & 26.9 \\
\hline \hline Subject 3 & 57.5 & 52.9 & 43.3 & 24.6 \\
\hline \hline Subject 4 & 57.4 & 49.05 & 44.02 & 23.3 \\
\hline \hline
\end{tabular}

\section{CONCLUSiON}

In this paper, we have proposed the ANFIS Link Quality Estimator (A-LQE) to develop an accurate model for RSSI prediction in on-body channels. The proposed model achieved accurate estimation of the target value, and incorporates for the first time the impact of body characteristics and body position as input parameters. The proposal has been trained and tested in a broad human sample and applied in a hybrid transmission power control policy that achieves significant energy savings.

\section{ACKNOWLEDGMENT}

The authors would like to thank to COLCIENCIAS and to Universidad Nacional de Colombia for their support in the development of this work. This work has been also partially supported by the Spanish Ministry of Economy and Competitiveness under contracts TIN2008-00508 and TEC2012-33892.

\section{REFERENCES}

[1] C. F. García-Hernández, P. H. Ibarguengoytia-Gonzalez, J. García-Hernández, and J. A. Pérez-Díaz, "Wireless sensor networks and applications: a survey," IJCSNS International Journal of Computer Science and Network Security, vol. 7, no. 3, pp. 264-273, 2007.

[2] A. Dhamdhere, V. Sivaraman, V. Mathur, and S. Xiao, "Algorithms for transmission power control in biomedical wireless sensor networks," in Asia-Pacific Services Computing Conference, 2008. APSCC'08. IEEE. IEEE, 2008, pp. 11141119.

[3] S. Xiao, A. Dhamdhere, V. Sivaraman, and A. Burdett, "Transmission power control in body area sensor networks for healthcare monitoring," Selected Areas in Communications, IEEE Journal on, vol. 27, no. 1, pp. 37-48, 2009.
[4] F. Di Franco, C. Tachtatzis, B. Graham, M. Bykowski, D. C. Tracey, N. F. Timmons, and J. Morrison, "The effect of body shape and gender on wireless body area network on-body channels," in Antennas and Propagation (MECAP), 2010 IEEE Middle East Conference on. IEEE, 2010, pp. 1-3.

[5] R. Fonseca, O. Gnawali, K. Jamieson, and P. Levis, "Fourbit wireless link estimation," in Proceedings of the Sixth Workshop on Hot Topics in Networks (HotNets VI), vol. 2007, 2007.

[6] M. Senel, K. Chintalapudi, D. Lal, A. Keshavarzian, and E. J. Coyle, "A kalman filter based link quality estimation scheme for wireless sensor networks," in Global Telecommunications Conference, 2007. GLOBECOM'07. IEEE. IEEE, 2007, pp. 875-880.

[7] I. Fernández Anitzine, J. A. Romo Argota, and F. P. Fontán, "Influence of training set selection in artificial neural networkbased propagation path loss predictions," International Journal of Antennas and Propagation, vol. 2012, 2012.

[8] T. Liu and A. E. Cerpa, "Foresee (4c): Wireless link prediction using link features," in Information Processing in Sensor Networks (IPSN), 2011 10th International Conference on. IEEE, 2011, pp. 294-305.

[9] Q. Tang, N. Tummala, S. K. Gupta, and L. Schwiebert, "Communication scheduling to minimize thermal effects of implanted biosensor networks in homogeneous tissue," Biomedical Engineering, IEEE Transactions on, vol. 52, no. 7, pp. 1285-1294, 2005.

[10] S. Kim and D.-S. Eom, "Rssi/lqi-based transmission power control for body area networks in healthcare environment," 2012.

[11] M. Vallejo, J. Recas, P. G. del Valle, and J. L. Ayala, "Accurate human tissue characterization for energy-efficient wireless on-body communications," Sensors, vol. 13, no. 6, pp. 7546-7569, 2013.

[12] I. Research, "Shimmer wbsn platform." http://www.shimmerresearch.com.

[13] C. Corporation, "Cc2420 2.4 ghz ieee 802.15.4 / zigbee-ready rf transceiver." http://www.ti.com/lit/gpn/cc2420.

[14] "Bc-601f fitscan segmental body composition monitor," http://www.tanita.com/en/bc601f/.

[15] J.-S. Jang, "Anfis: adaptive-network-based fuzzy inference system," Systems, Man and Cybernetics, IEEE Transactions on, vol. 23, no. 3, pp. 665-685, 1993.

[16] R. Jang, C. Sun, and E. Mizutani, "Neuro-fuzzy and soft computation," 1997.

[17] T. Nuguyen, C. Walker, and E. Walker, "A first course in fuzzy and neural control," Boca Raton, FL, 2003.

[18] M. Sugeno, Industrial applications of fuzzy control. Elsevier Science Inc., 1985.

[19] S. L. Chiu, "Selecting input variables for fuzzy models," Journal of Intelligent and Fuzzy Systems-Applications in Engineering and Technology, vol. 4, no. 4, pp. 243-256, 1996.

[20] M. Hosoz, H. Ertunc, and H. Bulgurcu, "Performance prediction of a cooling tower using artificial neural network," Energy Conversion and Management, vol. 48, no. 4, pp. 1349-1359, 2007.

[21] T. Nazmy, H. El-Messiry, and B. Al-Bokhity, "Adaptive neuro-fuzzy inference system for classification of ecg signals," in Informatics and Systems (INFOS), 2010 The 7th International Conference on. IEEE, 2010, pp. 1-6. 